\title{
ESTUDO DA CINÉTICA DE REMOÇÃO DE RESÍDUOS NA ETAPA DE ENXÁGUE DE SISTEMA DE LIMPEZA CIP
}

\author{
B. P. Bicalho ${ }^{1}$, L. Kunigk ${ }^{2}$, U. C. Filho ${ }^{1}$ e R. Gedraite ${ }^{1}$ \\ ${ }^{1}$ Universidade Federal de Uberlândia, Faculdade de Engenharia Química \\ ${ }^{2}$ Centro Universitário do IMT, Coordenadoria de Pós-graduação \\ E-mail para contato: barbarapbicalho@gmail.com
}

\begin{abstract}
RESUMO -Atualmente, a grande maioria dos processos de limpeza CIP é conduzida com base em procedimentos que levam em consideração a experiência dos operadores de processo no que tange ao tempo de funcionamento do ciclo. Este trabalho aborda o comportamento do sistema CIP em resposta a estímulos na vazão de operação do sistema, usando um trocador de calor. A cinética da remoção foi avaliada com base na variação do valor do $\mathrm{pH}$ medido. A partir das respostas obtidas, foram identificados os modelos do tipo FOPDT representativos da dinâmica do processo estudado para diferentes condições operacionais, que foram validados por meio de comparação com os dados experimentais disponíveis, sugerindo representatividade adequada e coerente. Os modelos foram usados para simular o comportamento da planta em estudo permitindo a avaliação da influência da oscilação nos valores da vazão de água de enxágue, em torno do ponto de operação, sobre o $\mathrm{pH}$. O desvio médio verificado foi inferior a $3 \%$, sugerindo deste modo que o modelo da planta pode ser usado para a avaliação in situ da calibração do elemento sensor empregado.
\end{abstract}

\section{INTRODUÇÃO}

A quantidade de água gasta nos processos de limpeza, em especial nos processos CIP, vem aumentando muito a cada ano, em decorrência da intensificação da produção de alimentos. Por se tratar de tema definido pelos órgãos de vigilância sanitária, não são poupados esforços para assegurar que o processo de limpeza dos equipamentos seja adequado e aderente aos critérios estabelecidos na legislação em vigor. Entretanto, este fato tradicionalmente implica na utilização, por parte da comunidade industrial, de maior quantidade de insumos do que aqueles que seriam necessários e tecnicamente suficientes para atender aos requisitos técnicos estabelecidos nas leis aplicáveis (BANSAL; CHEN, 2006).

Por serem procedimentos que requerem paradas de produção, os processos de higienização, muitas vezes, são realizados de forma negligenciada pelas empresas. Portanto, é de fundamental importância que sejam estudados e otimizados, através do estabelecimento das cinéticas de remoção de resíduos de cada etapa do processo(GEDRAITE et al., 2013). 
O estabelecimento do tempo adequado ao processo de higienização é fundamental para a eficiência do processo. Deve ser suficientemente longo para que as reações químicas e as interações físicas ocorram a contento; mas não deve ser excessivo, pois reduziria a produtividade da indústria (CARLOTTI, 2008).

Um processo de higienização é composto pelas seguintes etapas: pré-lavagem, circulação de solução detergente, enxágue e circulação de solução com ação antimicrobiana (sanificante). As etapas estudadas serão: aplicação de solução do detergente e o enxágue. A primeira consiste na ação do detergente sobre os resíduos para que estes sejam removidos da superfície dos equipamentos. O enxágue se caracteriza pela passagem de água no equipamento com função de remoção do detergente químico utilizado e dos resíduos deslocados pelo mesmo.

Dois parâmetros são tipicamente envolvidos em processos desta natureza, a saber: vazão de escoamento e temperatura. O primeiro está relacionado com a ação cisalhante proporcionada pela solução de detergente e água de enxágue sobre a superfície, enquanto o segundo permite melhor solubilização dos resíduos, facilitando sua remoção.Em um sistema de limpeza CIP, o tempo de escoamento das soluções, em cada etapa do processo de higienização, é o parâmetro de maior facilidade de manipulação e, por isso, o fator preferido de redução. Porém, se alterado de forma não criteriosa pode ocasionar a não efetividade do processo (GORMEZANO, 2007).

O equipamento utilizado neste trabalho foi um trocador de calor do tipo casco e tubos, de difícil higienização, pois as temperaturas envolvidas durante seu funcionamento acarretam, entre outros, na desnaturação proteica que dificulta a retirada dos resíduos, obrigando a realização de paradas mais frequentes (GEDRAITE et al., 2011).

A prática de higienização de equipamentos pela indústria de alimentos baseia-se em experiências empíricas, deixando o setor à mercê de informações provenientes dos fornecedores de detergentes e sanitizantes. Parâmetros semi-empíricos são adotados a partir de resultados finais de higienização considerados satisfatórios. A complexidade dos fenômenos existentes nas reações de remoção requer o aumento de trabalhos científicos que possam contribuir com os procedimentos adotados industrialmente (OLIVEIRA et al.,2012).

\section{MATERIAIS E MÉTODOS}

Na Figura 1 é apresentada a planta-piloto utilizada neste trabalho, a qual foi operada em regime descontínuo, e formada basicamente pelos seguintes elementos: (i)- tanque principal em aço inox com capacidade de aproximadamente $250 \mathrm{~L}$, (ii)- trocador de calor do tipo casco e tubos em aço inox, (iii)- bomba de deslocamento positivo para movimentação do produto alimentício, (iv)- bomba centrífuga para a movimentação do agente de aquecimento, reservatório cilíndrico vertical para armazenamento da água de aquecimento (SALVAGNINI; GEDRAITE, 2001).

O sistema eletrônico de aquisição de dados e controle do processo é apresentado pelo fluxograma da Figura 2. A plataforma responsável pela aquisição de dados e controle da planta em estudo é composta por um SECD (Sistema Eletrônico de Coleta de Dados), 
funcionando como um computador de processo e compreendendo basicamente as interfaces e um sistema computacional que, além do controle, proporcionam a aquisição e armazenamento dos dados de interesse. Este sistema é formado pelos seguintes itens: (i)- um microcomputador do tipo PC usado para a coleta e armazenamento dos dados de processo, (ii)- aplicativo LabVIEW®; (iii)- placa de aquisição de dados modelo NI PCI6259.; (iv)- quatro condicionadores de sinais para compatibilizar os sinais provenientes dos elementos sensores de temperatura; (v)- quatro elementos sensores de temperatura do tipo Pt 100; (viii)- medidor de vazão do tipo engrenagens ovais; (ix)- um conversor de sinal de corrente elétrica para pressão; (x)- uma válvula de controle pneumática e (xi)- um medidor de pH marca MICRONAL modelo B474.

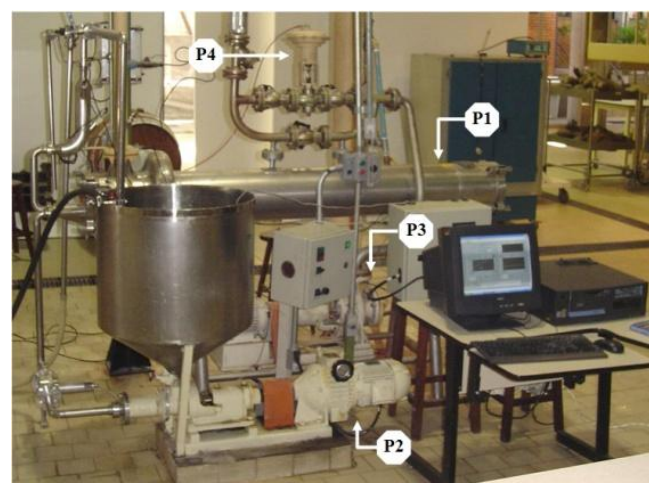

Figura 1 - Planta piloto utilizada.

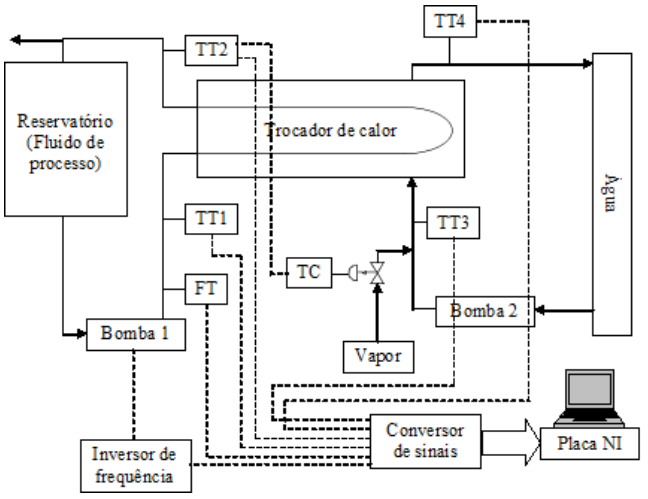

Figura 2 - Fluxograma de instrumentação do sistema.

A seguir é descrito de maneira resumida o procedimento experimental utilizado no desenvolvimento deste trabalho (CHEN et al., 2004) .

Inicialmente circulou-se leite à $80^{\circ} \mathrm{C}$ no interior do feixe tubular do trocador de calor com objetivo de causar a incrustação desejada para a etapa posterior de limpeza química. O leite foi deixado escoar por 90 minutos, com temperatura e vazão controladas em $80^{\circ} \mathrm{C}$ e $9,0 \mathrm{~L} \cdot \mathrm{min}^{-1}$, respectivamente. Finda esta etapa, foi realizada a drenagem de todo o leite que se encontrava no interior dos tubos do trocador de calor, tendo o mesmo sido deixado em repouso por aproximadamente uma hora para a consolidação do processo de incrustação nas paredes internas dos tubos que compõe o feixe tubular do mesmo.

Na sequência, foi iniciada a etapa de limpeza com solução de detergente nos tubos internos do trocador de calor. Primeiramente - enquanto se aguardava o tempo estipulado para a consolidação da incrustação - foi providenciada a preparação da solução detergente, composto por $\mathrm{NaOH}$ a $0,5 \mathrm{~g} \cdot \mathrm{L}^{-1}$. Após preparada, esta solução foi colocada em circulação nos tubos internos do trocador de calor, com temperatura e vazão controladas respectivamente em $50^{\circ} \mathrm{C}$ e $9,0 \mathrm{~L} \cdot \mathrm{min} .^{-1}$, durante uma hora, retornando a solução ao tanque de hidróxido de sódio quente. O escoamento da solução detergente foi mantida pelo período de uma hora, monitorando-se os valores dinâmicos do pH, vazão e temperaturas da solução e do agente de aquecimento.

Uma vez concluída a etapa de limpeza com a solução detergente, é iniciado o processo de enxágue com água potável para remoção do detergente residual aderido às paredes dos tubos do feixe tubular e que foi utilizado na etapa anterior. $\mathrm{O}$ equipamento 
é colocado em regime estacionário de operação, alimentando-se o bocal de entrada do trocador com água pré-aquecida em torno de $50^{\circ} \mathrm{C}$, mantendo-se controladas a temperatura em $50^{\circ} \mathrm{C}$ e a vazão em $9,0 \mathrm{~L} \cdot \mathrm{min}^{-1}$, e direcionando a saída do fluído de processo à um tanque intermediário de pequenas dimensões utilizado para a medição do $\mathrm{pH}$ sem turbulência.

O procedimento experimental citado nos parágrafos anteriores foirepetido para valores distintos de vazão de água de enxágue $\left(9,0 \mathrm{~L} \cdot \mathrm{min}^{.-1}, 10,5 \mathrm{~L} \cdot \mathrm{min} .^{-1} \mathrm{e} 12,0 \mathrm{~L} \cdot \mathrm{min}\right.$. ${ }^{1}$ ). As grandezas dinâmicas do sistema registradas ao longo dos ensaios, foram: temperaturas, vazão e $\mathrm{pH}$.

A identificação não-paramétrica foi baseada nas curvas de resposta do processo quando excitados por sinais de entrada do tipo degrau, impulso ou senoidal. A partir dessas curvas, foram extraídos modelos aproximados, de baixa ordem, utilizados para descrever o comportamento dinâmico do processo (AGUIRRE, 2007). Neste trabalho, estes modelos foram utilizados para representar o comportamento dinâmico do $\mathrm{pH}$ em função do tempo, tendo-se optado por ajustar um modelo de $1^{\mathrm{a}}$ ordem com atraso de transporte. Eles empregam parâmetros típicos de funções de transferência, a saber: ganho $(\mathrm{Kp})$, atrasode transferência ( $\tau \mathrm{p})$ e atraso de transporte ou tempo morto $(\theta \mathrm{p})$. Foi utilizado o Método de Sundaresan e Krishnaswamy (CAMPOS; TEIXEIRA, 2007)para a determinaçãodos parâmetros do modelo,por apresentar melhores resultados no ajuste dos dados experimentais (GEDRAITE et al., 2010).

O modelo da planta em estudo foi desenvolvido de maneira a contemplar o cenário de flutuações no valor da vazão de água de enxágue, que tipicamente são verificadas nas instalações industriais. Neste contexto, o modelo levou em conta o ajuste automático dos valores dos parâmetros de acordo com a vazão empregada. Na Figura 3 é apresentado o diagrama de simulação típico, considerando o modelo de referencia identificado e o modelo do processo. Os valores dos parâmetros $\mathrm{Kp}$, $\tau \mathrm{p}$ e $\theta \mathrm{p}$ foram obtidos por interpolação, para cada vazão estudada, a partir dos dados experimentais obtidos.

As flutuações nos valores das vazões foram modeladas por meio de variação aleatória das mesmas em torno do valor ajustado, segundo uma distribuição normal, com média igual a zero e variância igual a $1 \mathrm{~L} \cdot \mathrm{min}^{-1}$. $\mathrm{O}$ valor assumido para a variância corresponde àquele tipicamente verificado em malhas de controle de vazão de acordo com a precisão dos elementos que a compõem. O sinal gerado pelo bloco de função responsável por produzir a flutuação do valor da vazão é somado ao sinal de vazão estudado, como mostrado na Figura 3.

\section{RESULTADOS OBTIDOS \& DISCUSSÃO}

Com base nos resultados experimentais obtidos, foram propostos modelos matemáticos aproximados do tipo FOPDT para o comportamento dinâmico das cinéticas de remoção de resíduos em função das vazões consideradas na etapa de enxágue, cujos parâmetros identificados são apresentados na Tabela 1 (GEDRAITE et al., 2011). 


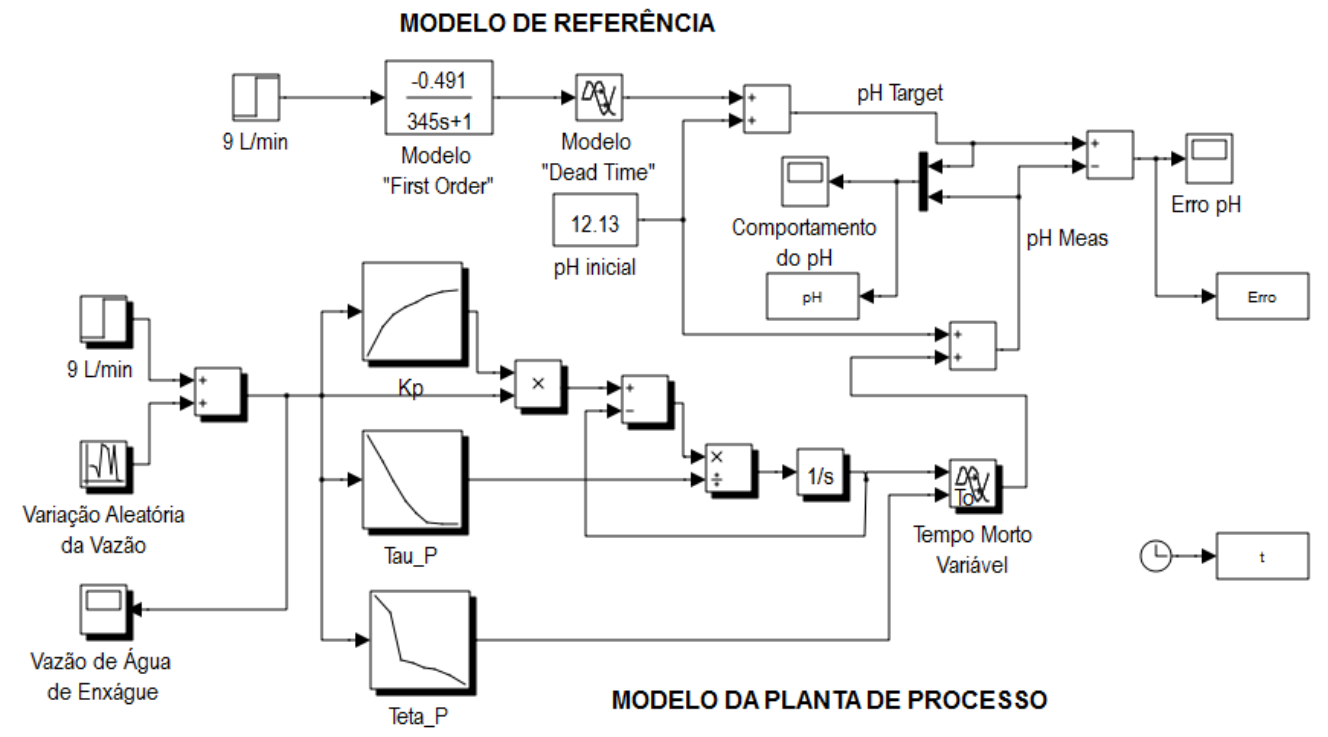

Figura 3 - Diagrama de simulação do processo em estudo.

Tabela 1 - Parâmetros dos modelos matemáticos FOPDT do processo estudado

\begin{tabular}{cccc}
\hline $\begin{array}{c}\text { Degrau } \\
{\left[\mathrm{L} \cdot \text { min. }^{-1}\right]}\end{array}$ & $\begin{array}{c}\text { Ganho, } \mathrm{K}_{\mathrm{p}} \\
{\left[\mathrm{pH} / \mathrm{L} \cdot \text { min. }^{-1}\right]}\end{array}$ & $\begin{array}{c}\text { Tempo morto, } \theta_{\mathrm{p}} \\
{[\mathrm{s}]}\end{array}$ & $\begin{array}{c}\text { Constante de tempo, } \tau_{\mathrm{p}} \\
{[\mathrm{s}]}\end{array}$ \\
\hline 9,0 & $-0,491$ & 453,0 & 345,0 \\
10,5 & $-0,421$ & 379,0 & 270,0 \\
12,0 & $-0,358$ & 360,5 & 214,5 \\
\hline
\end{tabular}

Nas Figuras 4, 5 e 6 são apresentados os resultados das simulações realizadas no aplicativo MATLAB®, para as vazões de 9,0 L.min. ${ }^{-1}, 10,5 \mathrm{~L} \cdot \mathrm{min}^{-1}$ e $12,0 \mathrm{~L} \cdot \mathrm{min}^{-1}$, utilizando o modelo de referencia ( $\mathrm{pH}$ target) e o modelo da planta de processo $(\mathrm{pH}$ meas).

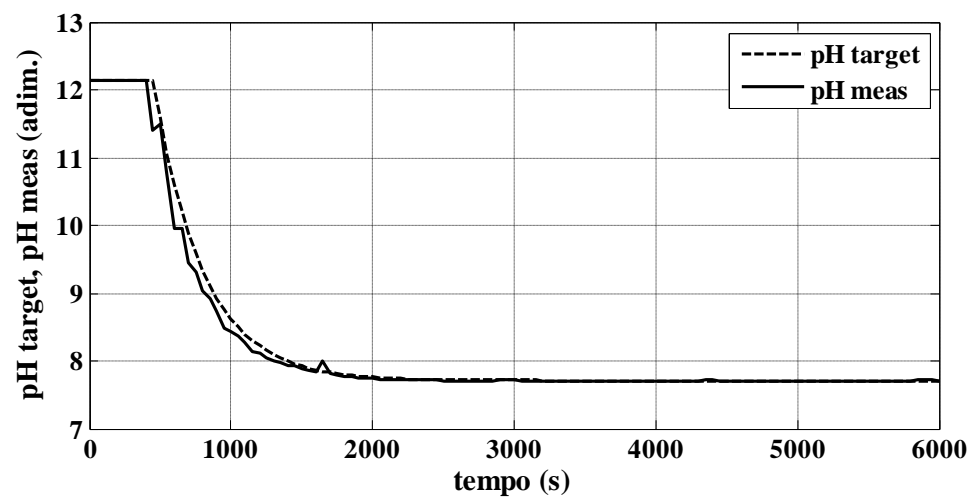

Figura 4 - Simulações dos modelos de referencia e da planta de processo $-9 \mathrm{~L} \cdot \mathrm{min}^{-1}$ 


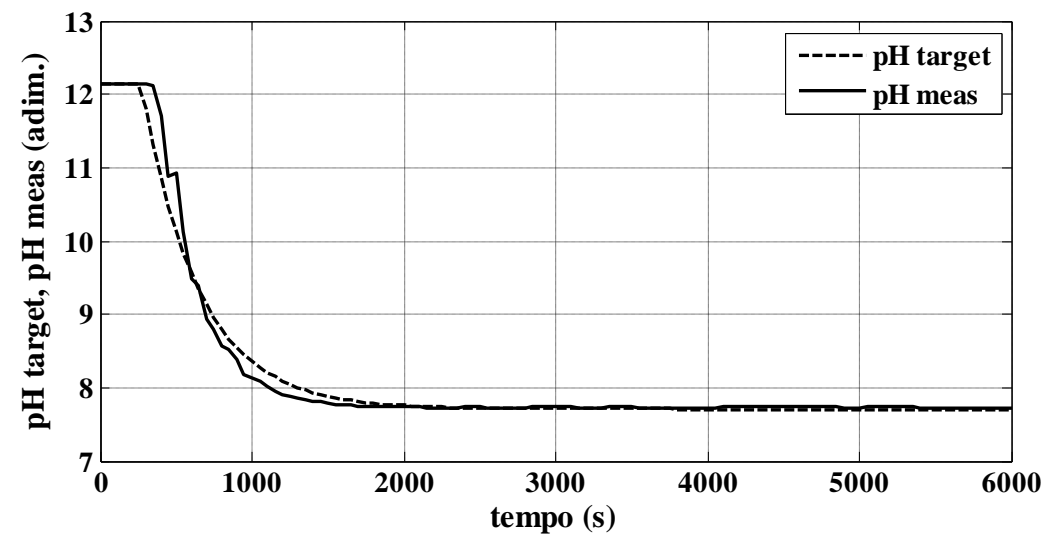

Figura 5 - Simulações dos modelos de referencia e da planta de processo $-10,5 \mathrm{~L} \cdot \mathrm{min}^{-1}$

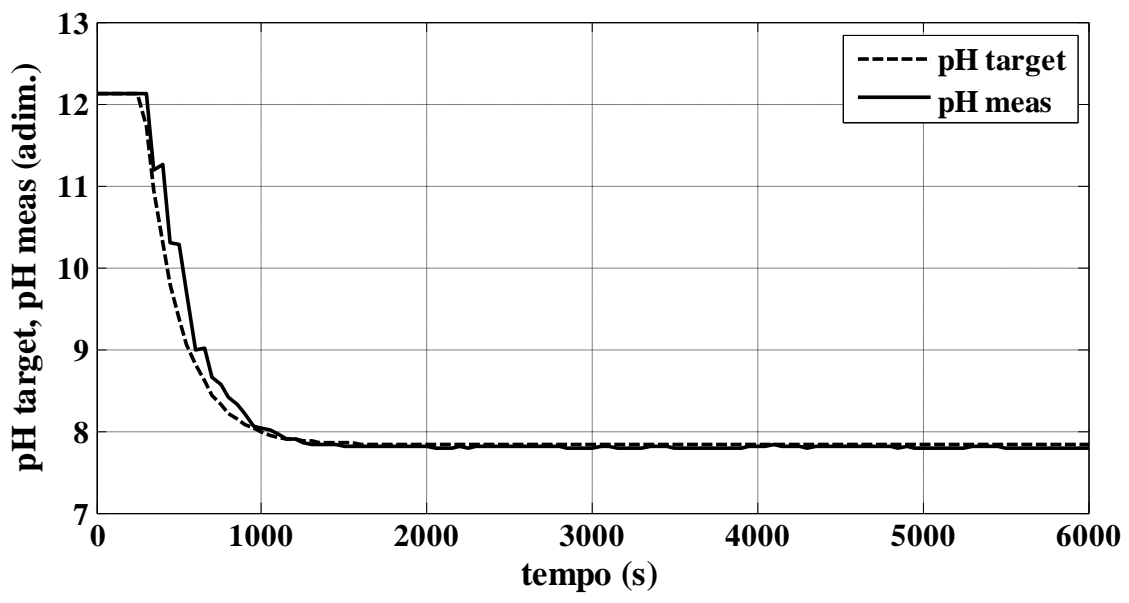

Figura 6 - Simulações dos modelos de referencia e da planta de processo $-12 \mathrm{~L} \cdot \mathrm{min}^{-1}$.

Com base nos resultados obtidos, pode-se constatar que a influência das perturbações nos valores das vazões sobre o comportamento temporal da variação do $p H$ measnão é significativa para as vazões consideradas, em que pese o fato da amplitude escolhida para o desvio $\left(1 \mathrm{~L} \cdot \mathrm{min}^{-1}\right)$ representar no primeiro caso aproximadamente $11 \%$ do valor de vazão usado, contra $9,5 \%$ para a vazão de $10,5 \mathrm{~L} \cdot \mathrm{min}^{-1}$ e $8 \%$ para $12 \mathrm{~L} \cdot \mathrm{min}^{-1}$.

$\mathrm{O}$ efeito das perturbações nos valores das vazões sugere que o comportamento temporal da variação do $p H$ meas para a vazão de $10,5 \mathrm{~L} \cdot \mathrm{min}^{-1}$ seja o mais adequado para se adotar como vazão de referencia, pois o $\mathrm{pH}$ measalcança o valor final residual em intervalo de tempo praticamente equivalente ao da vazão de $12,0 \mathrm{~L} \cdot \mathrm{min}^{-1}$. A explicação para este comportamento pode estar relacionada com o fato de não haver significativa alteração na turbulência promovida no escoamento entre os valores de vazão analisados.

Na Tabela 2 são apresentados os valores dos desvios máximos, mínimos e médios bem como os valores de desvio padrão - para os valores de erro de $\mathrm{pH}$. 
Tabela 2 - Desvios entre $p H$ target e $p H$ meas para as vazões estudadas

\begin{tabular}{|c|c|c|c|c|}
\hline \multirow[b]{2}{*}{$\begin{array}{c}\text { Degrau } \\
{\left[\mathrm{L} \cdot \min ^{-1}\right]}\end{array}$} & \multicolumn{4}{|c|}{ Desvios } \\
\hline & $\begin{array}{l}\text { Mínimo } \\
\text { [adim.] }\end{array}$ & $\begin{array}{c}\text { Máximo } \\
\text { [adim.] }\end{array}$ & $\begin{array}{l}\text { Médio } \\
\text { [adim.] }\end{array}$ & $\begin{array}{c}\text { Desvio padrão } \\
\text { [adim.] }\end{array}$ \\
\hline 9,0 & $-0,1432$ & 0,7301 & 0,0408 & 0,1099 \\
\hline 10,5 & -0.8462 & 0.258 & -0.01143 & 0.1509 \\
\hline 12,0 & $-0,9637$ & 0,0559 & 0,0288 & 0,1546 \\
\hline
\end{tabular}

Com base nos resultados obtidos, pode-se constatar que o desvio padrão entre o $p H$ target e o $\mathrm{pH}$ meas foi menor para a vazão de $9 \mathrm{~L} \cdot \mathrm{min}^{-1}$ e praticamente equivalente para as vazões de $10,5 \mathrm{~L} \cdot \mathrm{min}^{-1}$ e $12 \mathrm{~L} \cdot \mathrm{min}^{-1}$. Os desvios ocorreram de forma mais acentuada no início do processo de remoção dos resíduos. Este comportamento era esperado, pois é na fase inicial que a turbulência promove a maior remoção do $\mathrm{NaOH}$ residual.

\section{CONCLUSÕES}

Diante dos resultados apresentados, pode-se concluir que os modelosidentificados da planta revelam boa aderência com os comportamentos experimentais ensaiados, podendo ser usados como ferramenta de analise de processo. Com base neles, pode-se avaliar o impacto que alterações pré-estabelecidas no valor da vazão de água de enxágue apresentam sobre o tempo de operação e também sobre o volume total de água gasto em cada processo.

Com base nos resultados experimentais obtidos, pode-se verificar que quanto maior o valor de vazão de água de enxágue, menor é o intervalo de tempo necessário para que o sistema alcance a concentração mínima requerida de resíduo final, tendo sido observada uma redução de aproximadamente $50 \%$ no tempo de operação para um aumento no valor de vazão de $9 \mathrm{~L} \cdot \mathrm{min}^{-1}$ para $12 \mathrm{~L} \cdot \mathrm{min}^{-1}$. O efeito das oscilações aplicadas nos valores das vazões se revelou de maior influencia na cinética de remoção de resíduos para os valores mais elevados, sugerindo que os valores de $10,5 \mathrm{~L} \cdot \mathrm{min} .^{-1} \mathrm{e}$ $12 \mathrm{~L} \cdot \mathrm{min}^{-1}$ são praticamente equivalentes do ponto de vista experimental. $\mathrm{O}$ uso de 10,5 $\mathrm{L} \cdot \mathrm{min}^{-1}{ }^{-1}$ permite operar o sistema com menor gasto de água no sistema e aponta para a tendência de redução no consumo de água de enxágue em processos de limpeza CIP

\section{AGRADECIMENTOS}

Os autores agradecem à UFU, ao IMT e à FAPEMIG (Fundação de Amparo à Pesquisa do Estado de Minas Gerais) pelos recursos concedidos e apoio em pesquisas nos projetos No TEC-APQ-02100-12 (Estudo da Otimização de Sistema de Limpeza CIP) e Projeto de Participação Coletiva em Eventos Técnico-Científicos (PCE-0008214).

\section{REFERENCIAS}


AGUIRRE, L. A.Introdução à identificação de sistemas: técnicas lineares e não lineares aplicadas a sistemas reais.Belo Horizonte: Ed. UFMG, 2007

BANSAL, B.; CHEN, X. D.A critical review of milk fouling in heat exchangers.Vol. 5, $\mathrm{n}^{\circ}$. 2, pp. 27-33. 2006. Comprehensivereviews in foodscienceandfoodsafety.

CAMPOS, M.C.M.M; TEIXEIRA, H.C.G. Controles Típicos de Equipamentos e Processos Industriais. São Paulo: Ed. Edgard Blücher, 2007.

CARLOTTIFILHO, M. A. C. Estudo da cinética de remoção de resíduos de cálcio e de detergente alcalino nas etapas de pré-lavagem e de enxágue em um trocador de calor de placas. 2008. Dissertação de mestrado, CEUN-IMT.

CHEN, X. D.; OZKANY, N.; QIN, F.; XIN, H.; LIN, L.An effective CIP procedure for removing dairy protein based deposit - A Laboratory investigation. 2004. In: Proc. ofECI Conference on heat exchanger fouling and cleaning fundamentals and applications, Santa Fe, New Mexico, paper 44, 07p..

GEDRAITE, R.; KUNIGK, L.; RIBEIRO, S.; MELERO JR., V.; VASCONCELOS, F.; SISLIAN, R. Experimental investigation about the milk protein based deposit removal kinetics.2010. In: Proc. of World congress on communication and arts, WCCA, Guimarães/Portugal.

GEDRAITE, R., LOBATO, F. S., NEIRO, S. M. S., MELERO JR., V., AUGUSTO, S. R., KUNIGK, L. Modelagem matemática da cinética de remoção de resíduos em sistemas de limpeza CIP. 2011. In:Proc. of XXXII Iberian Latin-American Congress on Computational Methods in Engineering.

GEDRAITE, R., PINHEIRO, T. M. A., COUTINHO FILHO, U. KUNIGK, L, E NEIRO, S. M. S. Investigação da limpeza de depósitos proteicos na superfície de aço inox 316 por processo CIP com hidróxido de sódio. 2013. In: Anais do VII Congresso Brasileiro de Termodinâmica Aplicada (CBTermo).

GORMEZANO, L. Desenvolvimento e implementação de sistema para avaliar a cinética de remoção de resíduos presentes nos tubos de trocador de calor feixe tubular. Dissertação de mestrado, CEUN-IMT.2007.

OLIVEIRA, L. R., GEDRAITE, R.; COUTINHO FILHO, U. \& KUNIGK, L. Obtenção de modelo empírico para avaliação da quantidade de insumos gastos em um sistema CIP empregado na limpeza de um sistema de pasteurização de leite. 2012.XV Encontro de Modelagem Computacional e III Encontro de Ciência e Tecnologia de Materiais.

SALVAGNINI, W.; GEDRAITE, R. Rotina de Experimento para trocador de calor feixe tubular. São Caetano do Sul, SP: CEUN-IMT. 2001. 